\title{
Genipin-cross-linked collagen/chitosan biomimetic scaffolds for articular cartilage tissue engineering applications
}

\author{
Le-Ping Yan, ${ }^{1,2,3}$ Ying-Jun Wang, ${ }^{1,4}$ Li Ren, ${ }^{1,4}$ Gang Wu, ${ }^{1,4}$ Sofia G. Caridade, ${ }^{2,3}$ Jia-Bing Fan, ${ }^{5}$ \\ Ling-Yun Wang, ${ }^{1,4}$ Pei-Hong Ji, ${ }^{1}$ Joaquim M. Oliveira, ${ }^{2,3}$ João T. Oliveira, ${ }^{2,3}$ \\ João F. Mano, ${ }^{2,3}$ Rui L. Reis ${ }^{2,3}$
}

${ }^{1}$ School of Material Science and Engineering, South China University of Technology, Guangzhou 510641, People's Republic of China

23B's Research Group-Biomaterials, Biodegradables and Biomimetics, University of Minho, Headquarters of the European Institute of Excellence on Tissue Engineering and Regenerative Medicine, AvePark, Taipas, Guimarães 4806-909, Portugal

${ }^{3}$ IBB-Institute for Biotechnology and Bioengineering, PT Associated Laboratory, Guimarães, Portugal

${ }^{4}$ Key Laboratory of Specially Functional Materials, South China University of Technology, Ministry of Education,

Guangzhou 510641, People's Republic of China

${ }^{5}$ Department of Orthopedics, Third Affiliated Hospital of Sun Yat-sen University, Guangzhou, 510630,

People's Republic of China

Received 21 November 2009; revised 30 March 2010; accepted 4 May 2010

Published online 20 July 2010 in Wiley Online Library (wileyonlinelibrary.com). DOI: 10.1002/jbm.a.32869

\begin{abstract}
In this study, genipin-cross-linked collagen/chitosan biodegradable porous scaffolds were prepared for articular cartilage regeneration. The influence of chitosan amount and genipin concentration on the scaffolds physicochemical properties was evaluated. The morphologies of the scaffolds were characterized by scanning electron microscope (SEM) and cross-linking degree was investigated by ninhydrin assay. Additionally, the mechanical properties of the scaffolds were assessed under dynamic compression. To study the swelling ratio and the biostability of the collagen/chitosan scaffold, in vitro tests were also carried out by immersion of the scaffolds in PBS solution or digestion in collagenase, respectively. The results showed that the morphologies of the scaffolds underwent a fiber-like to a sheet-like structural transition by increasing chitosan amount. Genipin crosslinking remarkably changed the morphologies and pore sizes of the scaffolds when chitosan amount was less than $25 \%$. Either by increasing the chitosan ratio or performing
\end{abstract}

cross-linking treatment, the swelling ratio of the scaffolds can be tailored. The ninhydrin assay demonstrated that the addition of chitosan could obviously increase the cross-linking efficiency. The degradation studies indicated that genipin cross-linking can effectively enhance the biostability of the scaffolds. The biocompatibility of the scaffolds was evaluated by culturing rabbit chondrocytes in vitro. This study demonstrated that a good viability of the chondrocytes seeded on the scaffold was achieved. The SEM analysis has revealed that the chondrocytes adhered well to the surface of the scaffolds and contacted each other. These results suggest that the genipin-cross-linked collagen/chitosan matrix may be a promising formulation for articular cartilage scaffolding. (๑) 2010 Wiley Periodicals, Inc. J Biomed Mater Res Part A: 95A: 465-475, 2010.

Key Words: genipin, collagen, chitosan, articular cartilage, tissue engineering

\section{INTRODUCTION}

Articular cartilage (AC) has a limited capacity for self-regeneration upon damage caused by traumatic injuries or degenerative diseases. This feature of AC can be attributed to its characteristic avascular structure and relatively low cellular metabolic activity. ${ }^{1,2}$ Many techniques have been employed to treat articular cartilage defect, such as microfracture, multiple drilling and arthroscopic lavage with or without corticosteroids, and so on. ${ }^{3}$ But only a few of such treat- ments were able to achieve satisfactory clinical results as compared to autografts or allografts. ${ }^{4}$ Most of them had limited success to produce long-lasting hyaline cartilage. ${ }^{5}$ In recent years, tissue engineering has been introduced as a promising approach for the regeneration of damaged AC. $^{6}$ The basic principle of tissue engineering on AC is to seed the chondrocytes or differentiated stem cells in biocompatible and biodegradable scaffolds such as poly(lactic acid) or poly(glycolic acid), and then implant the cell/

Correspondence to: Y.-J. Wang; e-mail: imwangyj@scut.edu.cn or R. L. Reis; e-mail: rgreis@dep.uminho.pt

Contract grant sponsor: National Basic Research Program of China; contract grant number: 2005 CB623902

Contract grant sponsor: State Key Program of National Natural Science of China; contract grant number: 50732003

Contract grant sponsor: Natural Science Foundation Team Project of Guangdong; contract grant number: 4205786

Contract grant sponsor: Key Projects in the National Science and Technology Pillar Program in the Eleventh Five-year Plan Period; contract grant number: 2006BA116B04

Contract grant sponsor: Guangdong Natural Science Foundation; contract grant number: 07300602 
scaffold construct in the joint defect. Many efforts have been made to develop scaffolds with porous interconnected network structure and proper mechanical strength to support the cells' attachment, proliferation and differentiation toward repairing the AC defect. ${ }^{7,8}$

Many biomaterials, including naturally occurring and synthetic polymers or their combinations, have been extensively investigated as potential AC tissue engineering scaffolds both in vitro or in vivo. ${ }^{9-14}$ Among these biomaterials, collagen has received great attention, because it is the main component of AC specific extracellular matrix (ECM) which is reported to play important role in maintaining the chondrocytic phenotype and supporting the chondrogenesis both in vitro and in vivo. ${ }^{15}$ As the main constituent in $\mathrm{AC}$, collagen can maintain the chondrocyte phenotype and glycosaminoglycans (GAGs) production. ${ }^{16-18}$ Furthermore, collagen is known as a most promising biomaterial in tissue engineering due to its excellent biocompatibility and low antigenicity. ${ }^{19}$ However, the major drawbacks such as the rapid degradation and poor mechanical properties limit its further applications in tissue engineering. Cross-linking treatment or blending with other materials is an efficient method to overcome these constraints. Nowadays, there are two kinds of methods proposed in the cross-linking of collagen: chemical and physical methods. ${ }^{20}$ Although physical methods can avoid introduction of potential toxic residuals, they cannot yield high cross-linking degree. Therefore, the chemical cross-linking treatment is still the dominant choice. ${ }^{21-23}$ Numerous chemical reagents have been employed to crosslink collagen-based materials, including glutaraldehyde, formaldehyde, diisocyanate, diepoxide, and 1-ethyl-3-(3dimethylaminopropyl) carbodiimide. ${ }^{22,24,25}$ Recently, a naturally occurring cross-linking reagent genipin has received an increasing interest in biomedical applications. ${ }^{26,27}$ Extensive studies have been performed by Sung's group and they found that genipin not only has exhibited low cytotoxicity as compared to glutaraldehyde and epoxy, but also was able to efficiently cross-link cellular tissues and biomaterials containing primary amino groups. ${ }^{28-30}$ The use of genipin in cross-linking collagen for $\mathrm{AC}$ tissue engineering could be an attractive alternative to the traditional cross-linking reagents.

On the other hand, chitosan is also a natural and biodegradable polymer and has been widely applied in various biomedical applications, such as wound healing, cartilage and bone regeneration and drug delivery. ${ }^{31,32}$ Chitosan and glycosaminoglycans (GAGs) share some structural characteristics. $^{33}$ Taking into account the important roles of GAGs in stimulation of chondrogenesis, it seems a logical strategy to fabricate scaffolds by using GAGs or GAG analogs for cartilage tissue engineering. In addition, the strong interactions between collagen and chitosan molecular chains can make them to form a stable complex and reach a molecular level miscibility. ${ }^{34}$ Moreover, chitosan can act as cross-linking bridge to increase the cross-linking efficiency of the collagen-based scaffolds due to the abundant amino groups in its main chains. $^{20}$ Based on the previous investigation, ${ }^{16,24,26}$ the mixture of collagen and chitosan can be a promising candidate matrix for AC scaffolding.
In this study, genipin-cross-linked collagen/chitosan porous scaffolds were prepared for AC regeneration applications. The influence of chitosan amount and different concentrations of genipin on the physicochemical properties of the scaffolds were investigated. The morphology and pore size, cross-linking degree, swelling capacity, in vitro enzyme degradation and dynamic mechanical properties of the prepared collagen/chitosan scaffolds were characterized. Finally, cytotoxicity of collagen/chitosan scaffolds was screened by culturing New Zealand White Rabbit chondrocytes into the scaffolds until 12 days in vitro. The viability and morphology of the chondrocytes were examined by performing an 3-(4,5-Dimethylthiazol-2-yl)-2,5-diphenyl tetrazolium bromide (MTT) assay and scanning electron microscope (SEM) analysis, respectively.

\section{MATERIALS AND METHODS}

\section{Materials}

Chitosan $\left(M_{\mathrm{v}}=1.0 \times 10^{5}\right.$ and deacetylation degree of 92.5\%) was purchased from Sanland-Chem International (China). Collagenase type I (125 U/mg) was purchased from Sigma. Genipin was purchased from Wako (Japan). Pepsin (3000 U/mg) was purchased from Amresco. All other reagents and solvents were analytical grade and used as received.

Collagen type I was digested from bovine tendon (from slaughterhouse) by pepsin as previously described with minor modifications. ${ }^{34,35}$ Briefly, the bovine tendon was defatted and freed from adhering noncollagen materials before cut into small pieces. After digestion in pepsin solution for $72 \mathrm{~h}$, the acetic acid $(0.5 \mathrm{M})$ dissolved collagen solution was separated by centrifugation. Then, the supernatant collagen solution was precipitated by adding $1 \mathrm{~mol} / \mathrm{L} \mathrm{NaCl}$ solution. The precipitate was dialyzed and re-dissolved in acetic acid. Collagen was purified by repeating the precipitation and dialysis procedures. Finally, the purified collagen was frozen at $-20^{\circ} \mathrm{C}$ and lyophilized in a freeze dryer (ALPHA 2-4, Christ, Germany).

\section{Preparation of collagen/chitosan scaffolds}

The dried collagen or chitosan was dissolved in $0.2 \mathrm{M}$ acetic acid solution to prepare a $0.6 \%(\mathrm{w} / \mathrm{v})$ solution, respectively. The collagen and chitosan composites were prepared by mixing the two solutions at different ratios: $1: 0 ; 9: 1 ; 3: 1$; 1:1 (collagen:chitosan). The composite solutions were poured into the 6-well plates and the solution volume was controlled to keep the height of the solution about $5 \mathrm{~mm}$ in each well. Then, the solutions were kept in a saline bath (30 wt \% calcium chloride) at $-20^{\circ} \mathrm{C}$ for $10 \mathrm{~h}$, and lyophilized in a freeze dryer (ALPHA 2-4, Christ, Germany) to obtain the porous scaffold. The collagen/chitosan scaffolds containing $0,10,25$, and $50 \%$ chitosan amount were named as Col, Col/Cht-10, Col/Cht-25, and Col/Cht-50, respectively.

To remove the residual acetic acid, the fabricated collagen/chitosan scaffolds were immersed in gradient ethanol solution ranging from $100 \%$ to $0 \%(100,80,50,30$, and $0 \%$ ) for $2 \mathrm{~h}$ in each concentration, respectively. Then, the scaffolds were placed in the 6-well cell culture plate with 
each scaffold occupying a single well. Different concentration of genipin solutions $(0.1,0.3$, and $0.5 \%, \mathrm{w} / \mathrm{v})$ were prepared by dissolving genipin in phosphate buffer saline solution (PBS, pH 7.4, Sigma). Then, $4 \mathrm{~mL}$ of the genipin solution was added into each well and maintained for $24 \mathrm{~h}$ at room temperature. Finally, the genipin-cross-linked collagen/chitosan scaffolds were obtained by washing with deionized water; the scaffolds were frozen at $-20^{\circ} \mathrm{C}$ and lyophilized once again.

\section{Morphology observation}

The cross-section morphology of the collagen/chitosan scaffolds were observed by scanning electron microscopy (30XL FEG, Philips, Netherlands). The samples were sputter-coated with a layer of gold before observation, as previously described elsewhere. ${ }^{2}$

\section{Cross-linking degree determination}

The cross-linking degree of the genipin-cross-linked collagen/chitosan scaffolds was determined by ninhydrin assay and was defined as the ratio of the consumed amino groups in the cross-linked samples to the free amino groups in the corresponding uncross-linked samples. ${ }^{35}$ The test samples were first lyophilized for $24 \mathrm{~h}$ and then weighed. Subsequently, the test sample was heated with ninhydrin solution for 20 minutes at $100^{\circ} \mathrm{C}$. The amount of free amino groups in the test sample was determined by the optical absorbance of the solution at $570 \mathrm{~nm}$ recorded with a spectrophotometer (Model UV-3802; Unico Corp., Shanghai, China) using glycine at different concentrations (1.0, 2.0, 3.0, 4.0, and $5.0 \mathrm{mg} / \mathrm{mL}$ ) as standard. After heating with ninhydrin, the amount of free amino groups in the tested samples is proportional to the optical absorbance of the solution.

\section{Swelling ratio test}

The swelling ratio test was carried out as follows. Dry scaffolds were weighed $\left(w_{0}\right)$ at first, and then hydrated in PBS for $3 \mathrm{~h}$ at room temperature. After remove the excess surface water with filter paper, the wet scaffolds were weighed $(w)$ again. The swelling ratio of the scaffolds was defined as the wet weight increase $\left(w-w_{0}\right)$ to the initial weight $\left(w_{0}\right)$. The uncross-linked scaffolds with different chitosan amount were set as the controls for the corresponding cross-linked scaffolds.

\section{In vitro collagenase degradation}

The biodegradability of the collagen/chitosan scaffolds was determined by incubating each sample in $1 \mathrm{~mL}$ PBS ( $\mathrm{pH} 7.4$ ) containing $100 \mu \mathrm{g}$ collagenase type I (12.5 units) at $37^{\circ} \mathrm{C}$ for $12 \mathrm{~h}$. The degradation was discontinued by adding 100 $\mu \mathrm{L} 0.2 \mathrm{M}$ ethylenediaminetetraacetic acid (EDTA) and cooling the assay mixture in an ice bath immediately. ${ }^{36}$ The resulting mixture was centrifuged at $3000 \mathrm{rpm}$ for 10 minutes. Then, the supernatant was hydrolyzed in $6 \mathrm{M} \mathrm{HCl}$ at $110^{\circ} \mathrm{C}$ for $24 \mathrm{~h}$. The ultraviolet spectroscopy absorbance of hydroxyproline was examined according to the Woessner method. ${ }^{37}$ The biodegradation degree is defined as the ratio of hydroxyproline content in the degraded matrix to that in uncross-linked matrices.

\section{Dynamic mechanical analysis (DMA)}

The viscoelastic measurements were performed using a TRITEC8000B DMA from Triton Technology (UK), equipped with the compressive mode. The measurements were carried out at $37^{\circ} \mathrm{C}$. Samples were cut in cylindrical shapes with $4 \mathrm{~mm}$ diameter and $3 \mathrm{~mm}$ thickness (measured accurately for each sample via a digital micrometer with precision of $0.001 \mathrm{~mm}$ before the test). Scaffolds were always analyzed by immersing in a liquid bath (PBS, pH 7.4, Sigma) placed in a Teflon ${ }^{\circledR}$ reservoir. Scaffolds were previously immersed in a PBS solution until equilibrium was reached (overnight). The geometry of the samples was then measured and the samples were clamped in the DMA apparatus and immersed in the PBS solution. After equilibration at $37^{\circ} \mathrm{C}$, the DMA spectra were obtained during a frequency scan between 0.1 and $15 \mathrm{~Hz}$. The experiments were performed under constant strain amplitude $(70 \mu \mathrm{m})$. A small preload was applied to each sample to ensure that the entire scaffold surface was in contact with the compression plates before testing and the distance between plates was equal for all scaffolds being tested. Three samples were used per condition.

\section{Isolation and culture of chondrocytes}

The isolation of the chondrocytes was performed according to the Guide for the Care and Use of Laboratory Animals setting by National Institute of Health standards.

Chondrocytes were isolated from the femoral condyles and femoral heads of New Zealand White rabbits by enzymatic digestion. Briefly, the cartilage was collected in slices using a scalpel. Then, the slices were subjected to collagenase type II (Sigma) digestion. ${ }^{10}$ The obtained chondrocytes were cultured in Dulbecco's modified Eagle's medium (DMEM) containing: 10\% fetal bovine serum (FBS), vitamin C, $100 \mathrm{U} / \mathrm{mL}$ penicillin-streptomycin, and $25 \mu \mathrm{g} / \mathrm{mL}$ L-ascorbic acid. Cultures were maintained in an incubator $\left(5 \% \mathrm{CO}_{2}\right.$ and $99 \%$ humidity) at $37^{\circ} \mathrm{C}$. The culture medium was changed every 3 days.

The second passage chondrocytes and the Col/Cht-50 (cross-linked by $0.3 \%$ genipin) scaffolds were used in present study. The scaffolds were punched into cylinders $(6 \mathrm{~mm}$ in diameter and $5 \mathrm{~mm}$ in height), and sterilized with epoxy ethane. The scaffolds were pre-wetted in the culture medium overnight and then put in the 48-well culture plate before cell seeding. Then, $100 \mu \mathrm{L}$ of the chondrocyte suspension were seeded per scaffold $\left(5 \times 10^{5}\right.$ cells/scaffold $)$. The scaffolds were gently compressed by forceps to allow the cells suspension to absorb into the inner part of the scaffold. The scaffolds were transferred to another 48-well culture plate after $4 \mathrm{~h}$ of cell seeding. The culture medium in the plate was changed in $24 \mathrm{~h}$ for the first time and changed every 3 days after the first change. Cultures were maintained in a humidified incubator with $5 \% \mathrm{CO}_{2}$ at $37^{\circ} \mathrm{C}$. 


\section{Cell morphology and viability assay}

To observe the chondrocytes adhesion and proliferation onto the scaffolds, the scaffolds were washed with PBS then fixed in $2.5 \%$ glutaraldehyde for $24 \mathrm{~h}$ at the end of the 12 days of culturing. The constructs were gradually dehydrated in a series of ethanol solutions, and then isoamyl acetate was used to completely eliminate the ethanol. Samples were dried in a critical point dryer (HCP-2, Hitachi, Japan) then sputter coated with gold before examination under the SEM (30XL FEG, Philips, Netherlands).

Cell viability was analyzed via 3-(4,5-Dimethylthiazol-2yl)-2,5-diphenyl tetrazolium bromide (MTT) assay every 3 days. At first, the culture medium was removed, subsequently $300 \mu \mathrm{L}$ MTT $(1 \mathrm{mg} / \mathrm{mL})$ solution and then $700 \mu \mathrm{L}$ of culture medium was added to the cells. After $4 \mathrm{~h}$, the MTT solution and the culture medium were removed, and then $700 \mu \mathrm{L}$ dimethylsulphoxide (DMSO) solution was added and kept for 10 minutes. In the end, the DMSO solution was transferred to the 96-well cell culture plate and measured by a microplate reader (Sunrise-Basic Tecan) at $492 \mathrm{~nm}$. The scaffolds without cells seeding were used as controls.

\section{Statistical analysis}

Experiments were repeated three times and results expressed as an mean \pm standard deviation. The swelling ratio, cross-linking degree, enzymatic degradation tests and MTT test were evaluated by one-way analysis of variance (ANOVA). A comparison between two means was analyzed using Tukey's test with statistical significance set at $p<0.05$.

\section{RESULTS AND DISCUSSION Morphology of scaffolds}

Figure 1 shows the cross-sectional morphology of the uncross-linked and genipin-cross-linked collagen/chitosan scaffolds with different chitosan amounts. All scaffolds possessed a three-dimensional interconnected macroporous structure. The fiber- and strip-like structure of collagen scaffold with pore size ranged from 100 to $200 \mu \mathrm{m}$ is presented in Figure 1(a). It is consistent with the triple-helix structure of collagen which imparts Col scaffold with the fibrous morphology. It can be found that the Col/Cht-10 and Col/Cht-25 scaffolds displayed a sheet-like structure instead of a fiberlike structure, and their pore size decrease to about 50-150 $\mu \mathrm{m}$ [Fig. 1(c,e)]. The explanation for this observation can be related with the fact that chitosan is a semi-crystal polymer and it tends to form the membrane structure. ${ }^{24}$ With low chitosan amount, the scaffolds would form many small pieces of sheets which lead to the slightly decrease in pore size. While being immersed in genipin solution, the collagen scaffold would be cross-linked and rehydrated at the same time. The latter procedure would lead to the collapse of the porous structure and the fusion of the fibers before genipin can play its role. For this reason, the collagen fiber formed a typical membrane-like structure and with pore size lower than $100 \mu \mathrm{m}$ after undergoing the cross-linking step [Fig. 1(b)]. The cross-linked Col/Cht-10 scaffold consisted of lattice-like structure with pore size between 100 and $150 \mu \mathrm{m}$
[Fig. 1(d)]. As the chitosan amount increased to 25\%, the lattices become ordered and its size increased with pore size ranging from $150 \mu \mathrm{m}$ to $200 \mu \mathrm{m}$ [Fig. 1(f)]. During the cross-linking procedure, scaffolds with higher chitosan content had more cross-linking points, so the small sheets would be linked together to form larger sheets and inducing the formation of larger pores after re-lyophilization.

It is noticed that honeycomb-like ordered structure with large pore walls and pore size ranged from 100 to $200 \mu \mathrm{m}$ were evident in the Col/Cht-50 scaffolds [Fig. 2(a)]. When chitosan amount increased to $50 \%$, the chitosan in the scaffolds can form continuous and large pieces of sheets which led to the observed larger pore wall structure and larger pore size compared to the uncross-linked Col, Col/Cht-10 and Col/Cht-25. There were no obvious changes in the pore size and morphology of the Col/Cht-50 scaffolds after crosslinking with different concentration of genipin [Fig. 2(b-d)]. Since chitosan possesses higher mechanical strength than collagen, the Col/Cht-50 scaffold can retain its original structure when cross-linked with genipin. For this reason no collapse and fusion of the pores was observed in the cross-linked scaffolds.

\section{Cross-linking degree}

Figure 3 displays the cross-linking degree of the collagen/chitosan scaffolds. The cross-linking degree ranged from 63.93 to $74.14 \%$ when cross-linked by $0.3 \%$ genipin. The crosslinking degree of Col/Cht-50 scaffolds ranged from 48.28 to $80.71 \%$ as genipin concentration increased from 0.1 to $0.5 \%$.

These results demonstrated that genipin is a favorable cross-linking reagent for collagen or/and chitosan, as it can efficiently cross-link the amino groups even at low concentration. Moreover, as the genipin concentration increased, more and more amino groups were cross-linked which led to a higher cross-linking degree.

Sung et $a l^{38}$ reported that the amino acid residues within collagen that can react with genipin were lysine, hydroxylysine and arginine. This group ${ }^{39}$ also showed that genipin can react with the amino groups in chitosan. Additionally, they both found that genipin can form intramolecular and intermolecular cross-linking networks. Therefore, during the cross-linking treatment, collagen and chitosan can cross-link with each other to form an interpenetrating polymer network (IPN). It is well know that the ratio of amino groups in chitosan molecular chain is much higher than that in collagen molecular chain. Thus, the free amino groups can act as the cross-linking points. As a result, the amount of cross-linking points directly relate to the original amount of free amino groups in the scaffolds. During the cross-linking step, more cross-linking points will lead to higher biostability. Therefore, we deduce that the addition of chitosan can improve the biostability of collagen. This issue will be further discussed in the following section.

\section{Swelling ratio test}

The water binding capacity of the scaffold plays an important role in tissue regeneration. The swelling ratio of scaffolds with different chitosan amount (uncross-linked and 

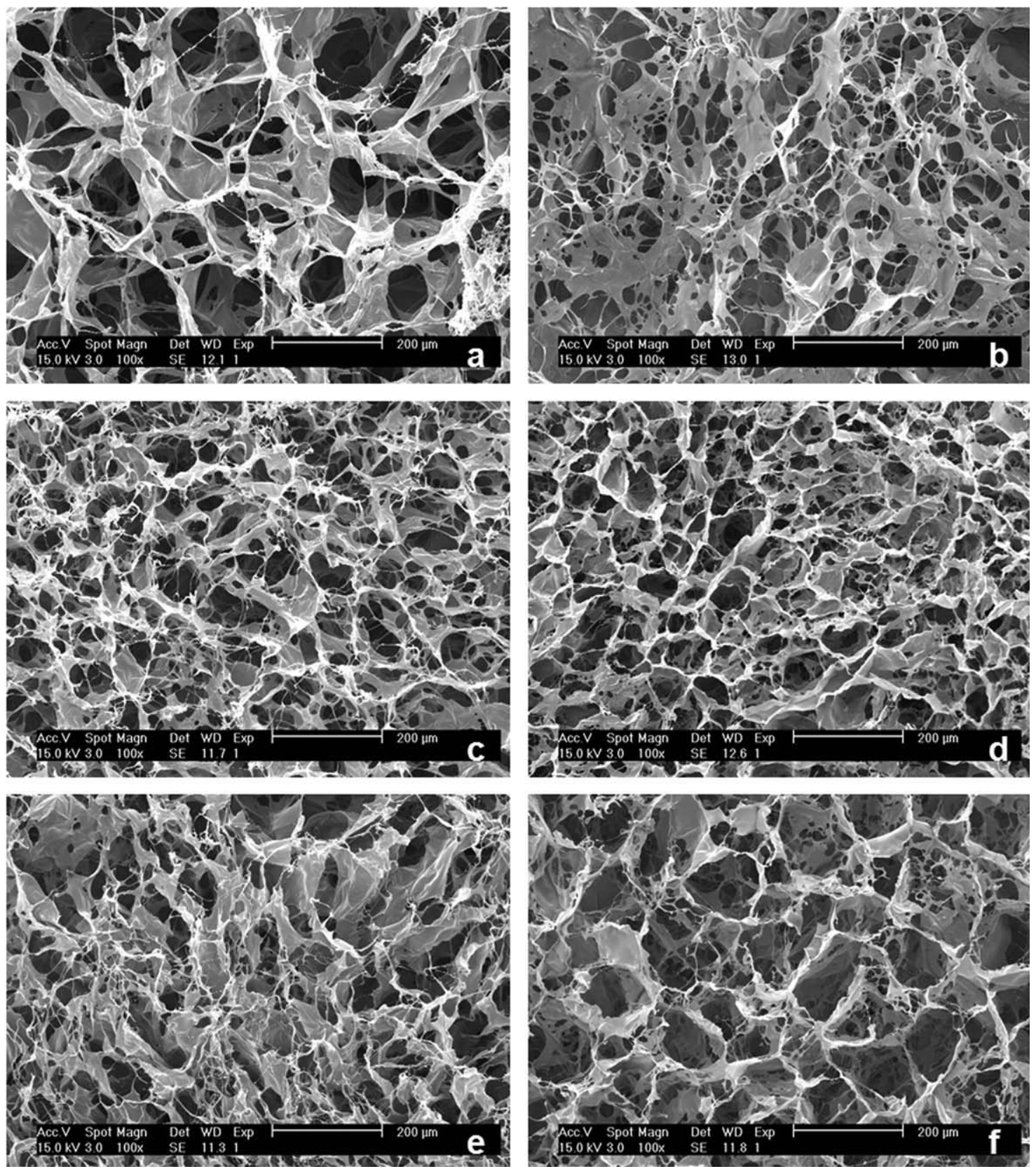

FIGURE 1. The cross-section SEM images of uncross-linked and $0.3 \%$ genipin cross-linked collagen/chitosan scaffolds. (a), (c), and (e) represent the morphology of the Col, Col/Cht-10, Col/Cht-25 scaffolds, respectively; (b), (d), and (f) display the morphology of the $0.3 \%$ genipin cross-linked collagen/chitosan scaffolds corresponding to (a), (c), and (e), respectively.

cross-linked) is shown in Figure 4. We could find that the water-retention ability of the scaffolds increased by increasing the chitosan amount, despite the cross-linked or the uncross-linked scaffolds. The cross-linked scaffolds had a higher swelling ratio as compared to the corresponding uncross-linked ones.

The swelling ratio of the scaffold strongly depends on the hydrophilic nature and microstructure of the scaffold. Since collagen and chitosan are both hydrophilic materials, the ability to retain the scaffold porous structure seems to be the main explanation for the differences observed in the swelling ratio. The poor mechanical properties of collagen led to the collapse of the porous structure when it was taken out from PBS solution. Contrarily, chitosan possesses higher elasticity which is helpful for the retention of the scaffold original porous structure. Hence, the swelling ratio of the scaffolds increased as the increase of chitosan amount in the scaffolds (Fig. 4).

It's well known that the swelling or hydrophilic property of the materials would decrease after cross-linking treatment, as the reduction of the hydrophilic groups (such as the amino or carboxylic groups) which are consumed during the cross-link reaction. However, in our case, the swelling ratio of the cross-linked scaffolds was higher than that of 

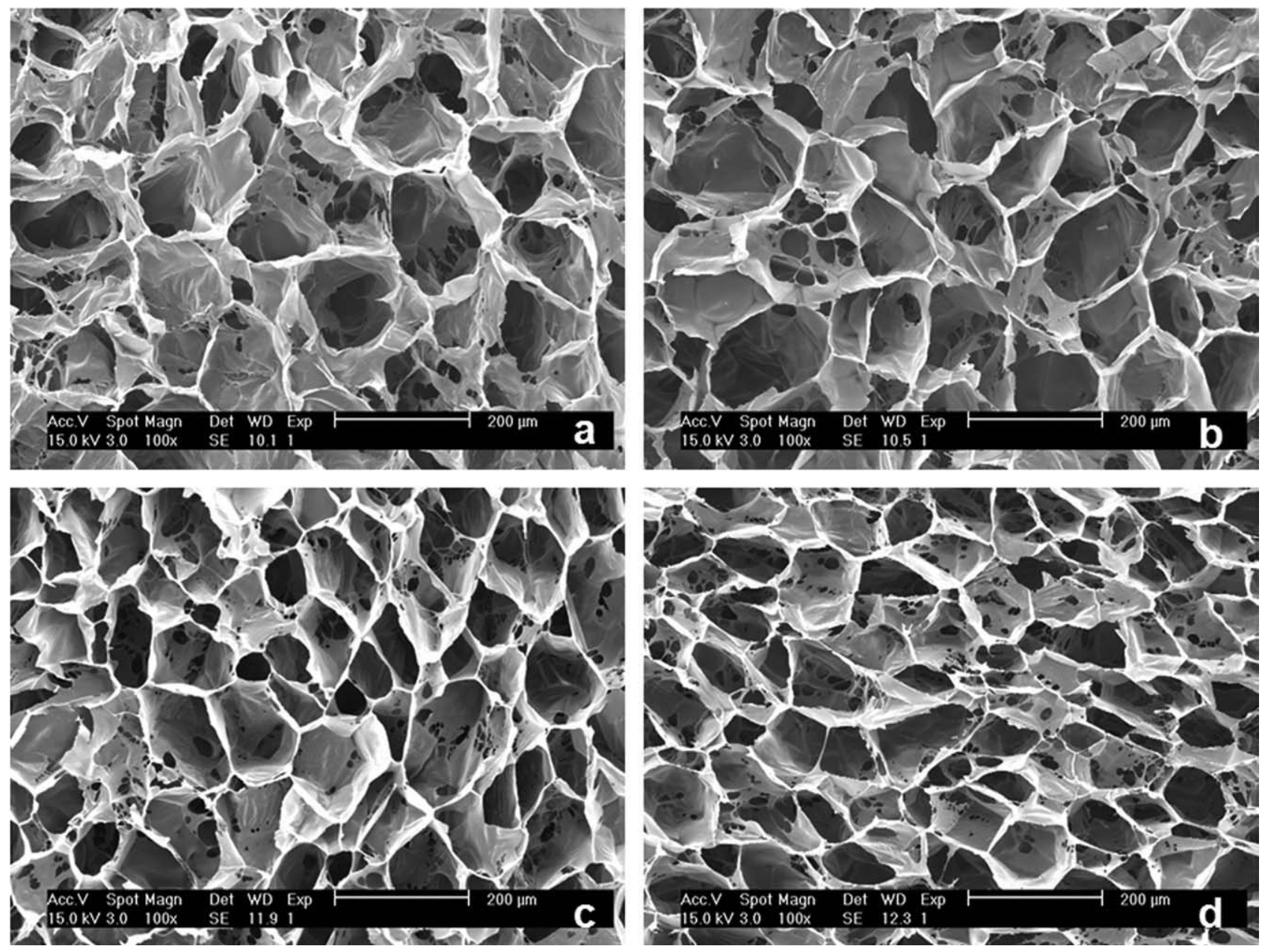

FIGURE 2. The cross-section SEM images of the Col/Cht-50 scaffolds cross-linked via different concentration of genipin. (a) Control; (b) $0.1 \%$ genipin; (c) $0.3 \%$ genipin; and (d) $0.5 \%$ genipin.

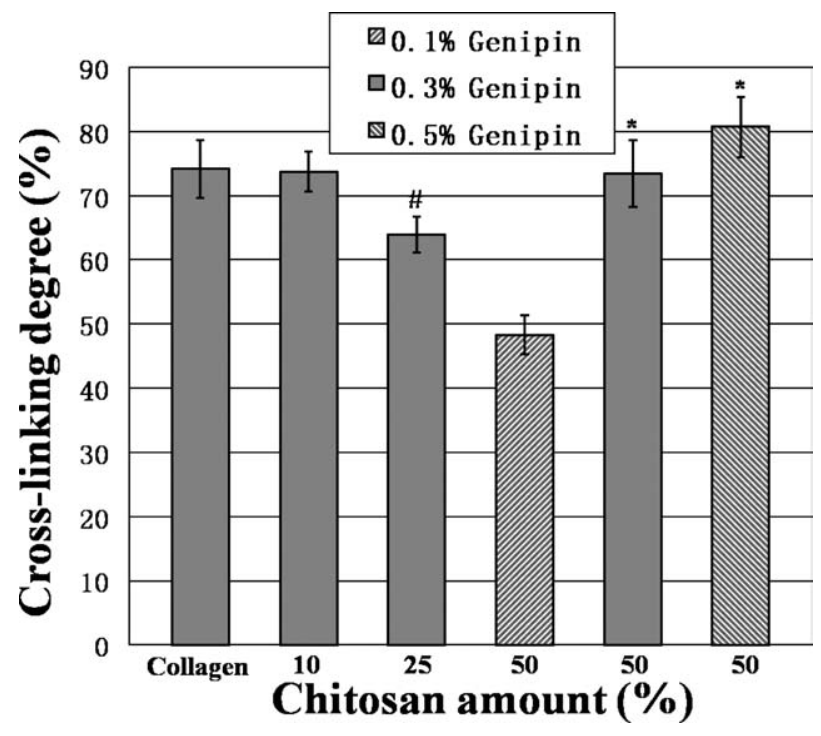

FIGURE 3. The cross-linking degree of collagen/chitosan scaffolds with different chitosan amount and being cross-linked via different genipin concentration. * indicates statistical significance when compared with $0.1 \%$ genipin cross-linked Col/Cht-50 scaffold $(p<0.05)$ and \# indicates statistical significance when compared with $0.3 \%$ genipin cross-linked collagen scaffold $(p<0.05)$.

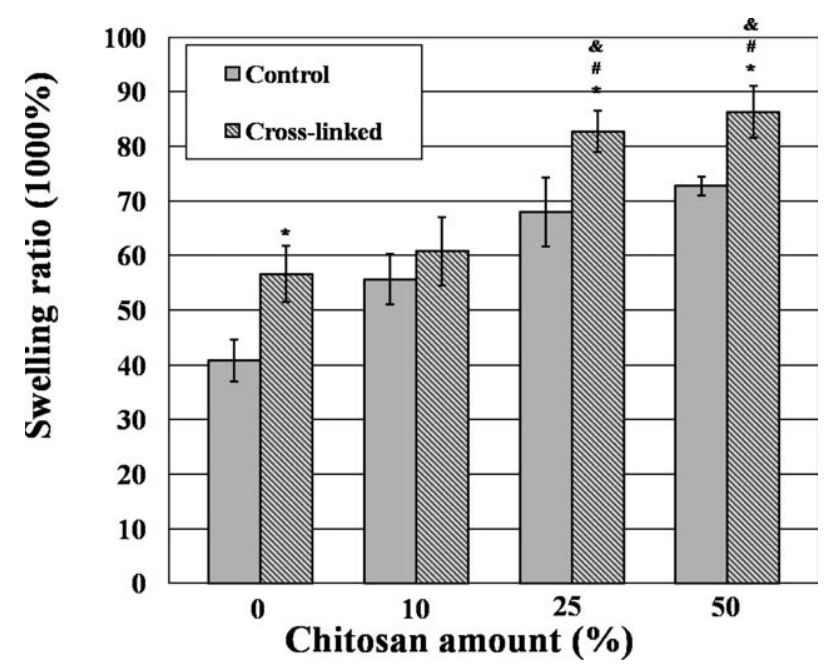

FIGURE 4. The swelling ratio of the uncross-linked and cross-linked ( $0.3 \%$ genipin) collagen/chitosan scaffolds with different chitosan amount. * indicates statistical significance when compared with the corresponding controls (uncross-linked scaffolds) $(p<0.05)$. \# and \& indicate statistical significance when compared with the cross-linked Col and Col/Cht-10 scaffolds, respectively $(p<0.05)$. 


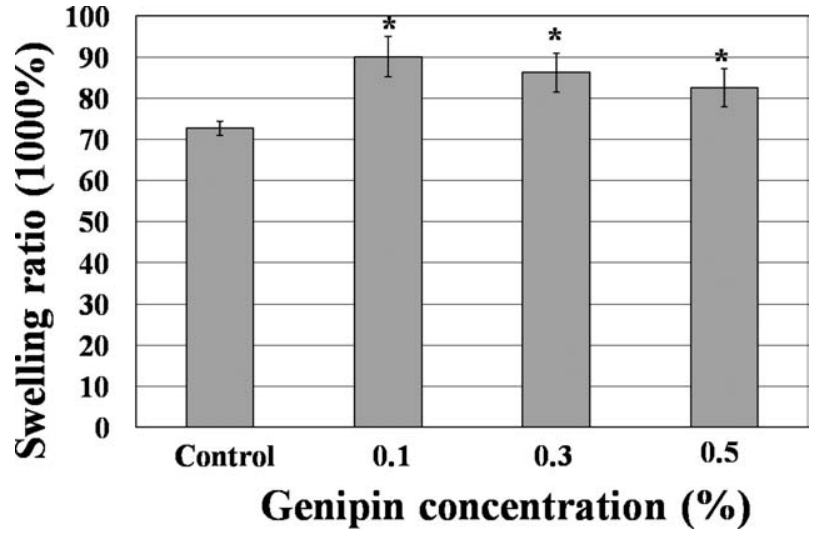

FIGURE 5. The swelling ratio of the Col/Cht-50 scaffolds cross-linked via different genipin concentration. * indicates statistical significance when compared with control (uncross-linked Col/Cht-50 scaffold) $(p<0.05)$.

the corresponding uncross-linked scaffolds (Fig. 4). Our explanation is that, under hydration conditions, the collapse of the porous structure was inevitable in the case of uncrosslinked scaffolds, but the cross-linking treatment can enhance the scaffolds' structural stability and subsequently increased the scaffold water-retention ability.

The swelling ratio of the Col/Cht-50 scaffolds seemed to slightly decrease as the genipin concentration increased from 0.1 to $0.5 \%$, but they were significant higher than that of the uncross-linked scaffolds (Fig. 5).

Genipin can efficiently cross-link the scaffold by the reaction with amino groups. ${ }^{27}$ The Col/Cht-50 scaffolds contain the highest ratio of amino groups as compared to the Col, Col/Cht-10 and Col/Cht-25 scaffolds. Therefore, the Col/Cht-50 scaffold cross-linked by genipin can keep its original porous structure, which led to the higher swelling ratio than the control. Since all the cross-linked Col/Cht-50 scaffolds presented similar morphology and pore size (Fig. 2) and favorable biostability (Fig. 6), we hypothesize that the hydrophilic groups left in the scaffold played the key role on the scaffolds swelling ability. But there were no significant differences in the swelling ratio of the Col/Cht-50 scaffolds cross-linked via different genipin concentration.

\section{In vitro biodegradability}

The in vitro enzymatic degradation properties of the collagen/chitosan scaffolds under uncross-linked or cross-linked treatment are shown in Figure 6. Collagen in all of the uncross-linked collagen/chitosan scaffolds were completely digested after incubated in collagenase type I solution for $12 \mathrm{~h}$. The addition of chitosan did not affect the degradation of collagen in the uncross-linked scaffolds.

After performing the cross-linking treatment with $0.3 \%$ genipin solution for $24 \mathrm{~h}$, the anti-degradation ability of collagen enhanced remarkably. There was only $10.71 \%$ degradation in the pure collagen scaffold. Furthermore, the addition of chitosan greatly improved the enzymatic stability of collagen. The degradation ratio of collagen in the scaffolds

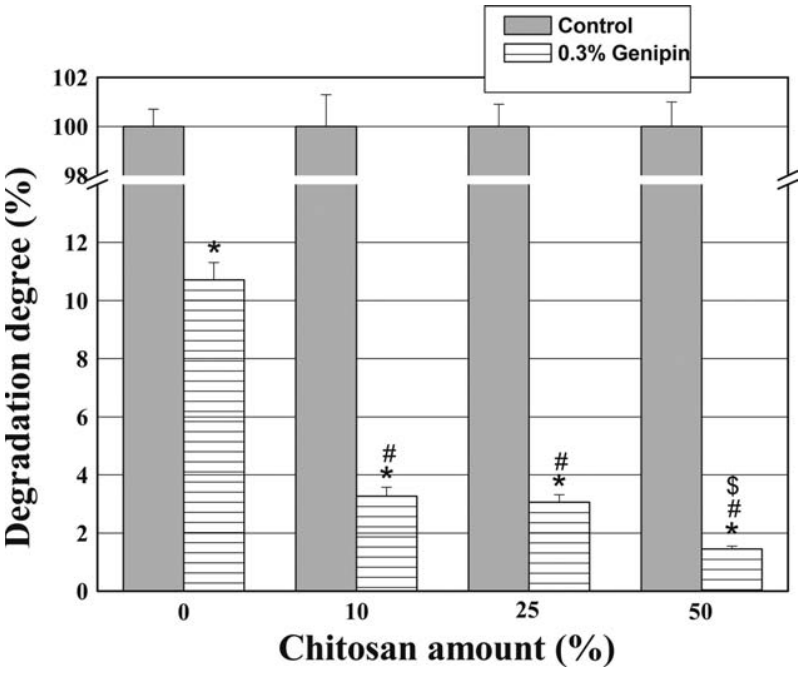

FIGURE 6. The degradation degree of the uncross-linked and crosslinked $(0.3 \%$ genipin) collagen/chitosan scaffolds with different chitosan amount after digestion in $100 \mu \mathrm{g} / \mathrm{mL}$ (12.5 units) collagenase type I for $12 \mathrm{~h} .{ }^{*}$ indicates statistical significance when compared with corresponding controls (uncross-linked scaffolds) $(p<0.05)$. \# indicates statistical significance when compared with cross-linked Col ( $p<$ $0.05)$. \$ indicates statistical signifsicance when compared with crosslinked Col/Cht-10 and Col/Cht-25 $(p<0.05)$.

decreased as increasing chitosan amount. The degradation ratio of collagen in Col/Cht-50 scaffold was only $1.45 \%$.

The influence of genipin concentration on the degradation of Col/Cht-50 scaffolds is shown in Figure 7. Collagen degradation decreased by increasing the genipin concentration. After cross-linking with $0.5 \%$ genipin, the degradation degree of collagen was below $1 \%$.

These results indicated that genipin can effectively cross-link collagen, thus improving its enzymatic stability; meanwhile the presence of chitosan can dramatically

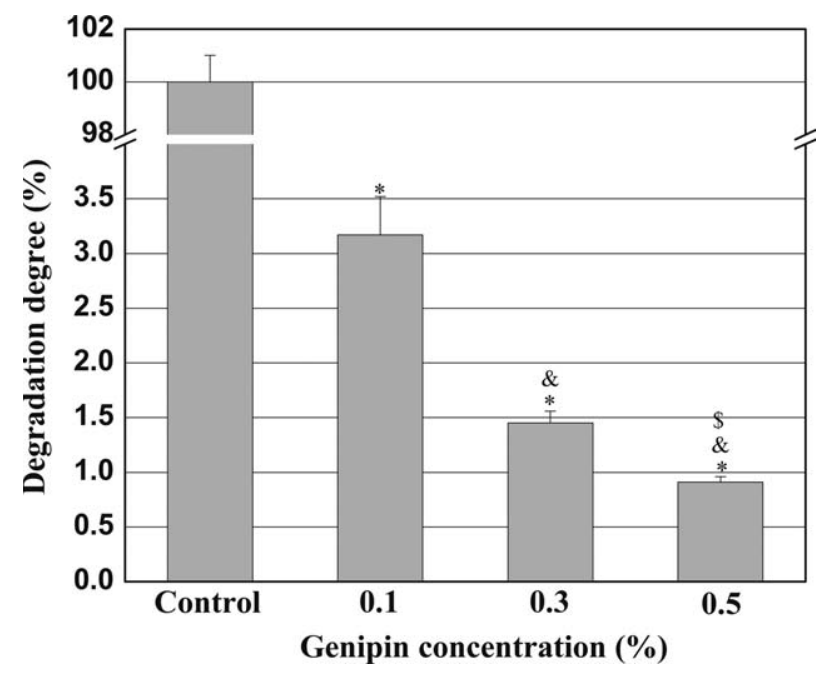

FIGURE 7. The degradation degree of the Col/Cht-50 scaffolds crosslinked via different genipin concentration after digestion in $100 \mu \mathrm{g} / \mathrm{mL}$ (12.5 units) collagenase type I for $12 \mathrm{~h} .{ }^{*}, \&$ and $\$$ indicate statistical significance when compared with control, $0.1 \%$ and $0.3 \%$ genipin cross-linked scaffolds, respectively $(p<0.05)$. 

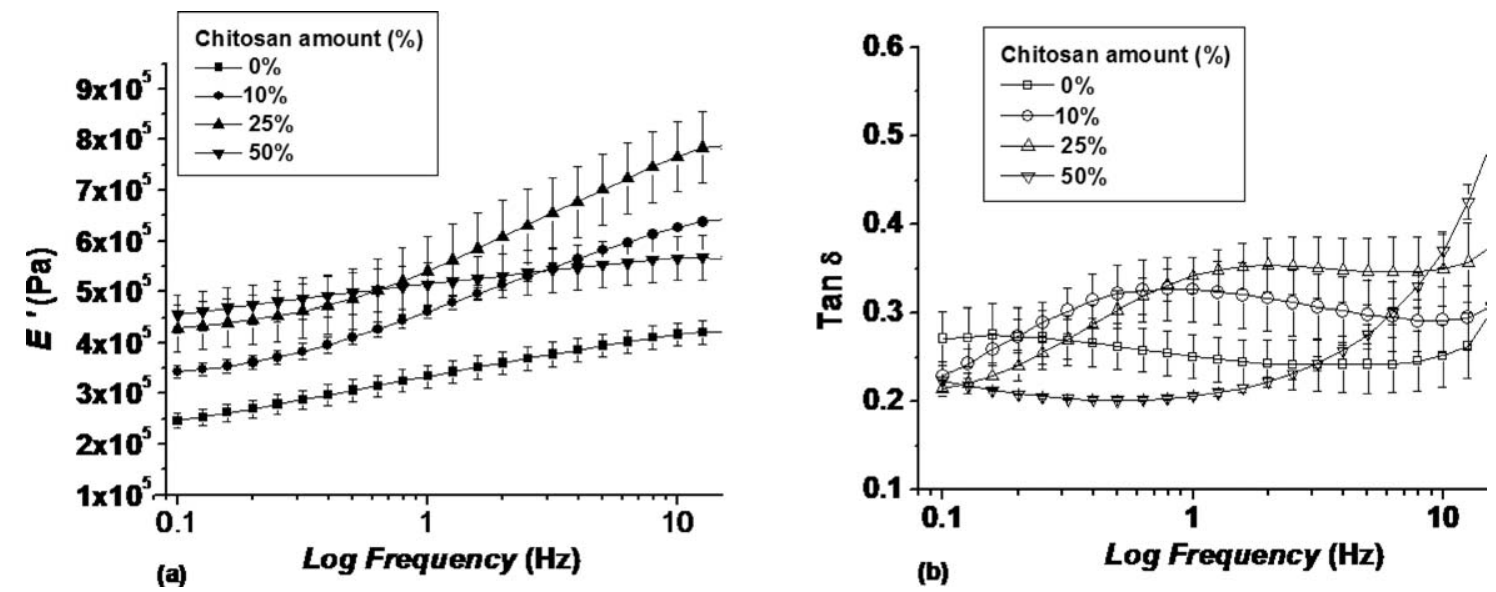

FIGURE 8. (a) Storage modulus $\left(E^{\prime}\right)$ and (b) loss factor (tan $\delta$ ) of $0.3 \%$ genipin cross-linked collagen/chitosan scaffolds with different chitosan amount measured by immersing the samples in PBS at $37^{\circ} \mathrm{C}$.

increase the cross-link efficiency. It has been shown ${ }^{40}$ that collagenase induces collagen degradation via cleaving the peptide bonds between leucine and glycine triple-helical collagen. The intramolecular and intermolecular cross-linking network on collagen or collagen/chitosan scaffolds hinders the collagenase to access the cleavage sites in collagen. For this reason the cross-linked scaffolds presented a higher biostability. On the other hand, the cross-linking sites depend on the amount of amino groups available. Therefore, the cross-linked Col/Cht-50 scaffolds displayed significant enhancement in collagen biostability compared to that of the cross-linked Col/Cht-10 and Col/Cht-50 scaffolds.

We also noticed an interesting phenomenon when correlating the biostability and the cross-linking degree data. The scaffolds with very different cross-linking degrees but shared nearly the same biostability. For example, the crosslinking degrees of the $0.1 \%$ genipin-cross-linked Col/Cht-50 scaffolds and the $0.3 \%$ genipin-cross-linked Col/Cht-10 scaffolds were 48.28 and $73.74 \%$, respectively (Fig. 3). However, the enzymatic degradation of these two groups was both about 3.2\% (Figs. 6 and 7). The explanation for this can be related to the different amount of original amino groups presented in these two groups' samples. The Col/Cht-50 scaffolds possess more original free amino groups than the Col/Cht-10 scaffolds. With no doubt, the abundant amino groups in Col/Cht-50 will bring about more cross-linking points even at low genipin concentration.

\section{Dynamic mechanical analysis (DMA) test}

Dynamic mechanical analysis (DMA) is an adequate tool to characterize the mechanical/viscoelastic properties of polymeric materials. It has been reported that ${ }^{41,42}$ polymeric materials present different behaviors depending on the kind of environment where they are tested. Since articular cartilage often bears a dynamic compression force, DMA experiments were performed in a hydrated environment and at $37^{\circ} \mathrm{C}$ to assess how scaffolds behave in more realistic conditions. ${ }^{42,43}$

Figure 8 presents the viscoelastic behavior of the scaffolds with different chitosan amount being all cross-linked by $0.3 \%$ genipin. The storage modulus $\left(E^{\prime}\right)$ of all the scaffolds tends to increase by increasing the frequency
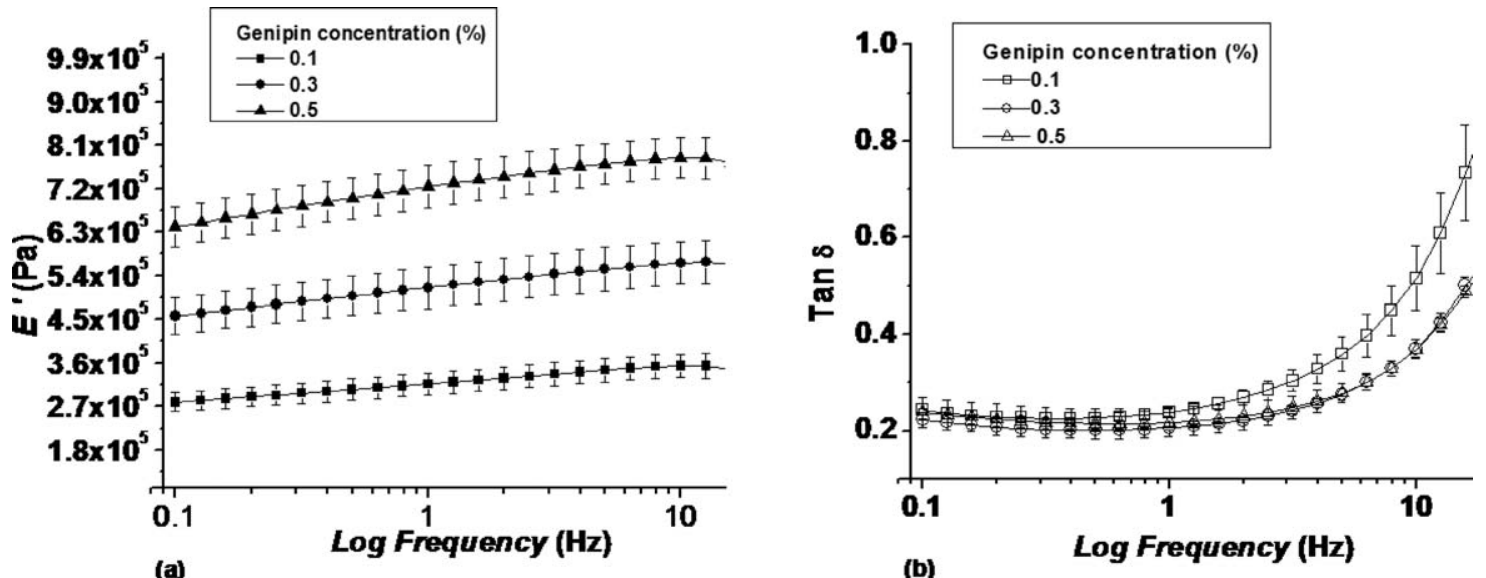

FIGURE 9. (a) Storage modulus $\left(E^{\prime}\right)$ and (b) loss factor (tan $\delta$ ) of Col/Cht-50 scaffolds cross-linked via different genipin concentration measured by immersing the samples in $\mathrm{PBS}$ at $37^{\circ} \mathrm{C}$. 


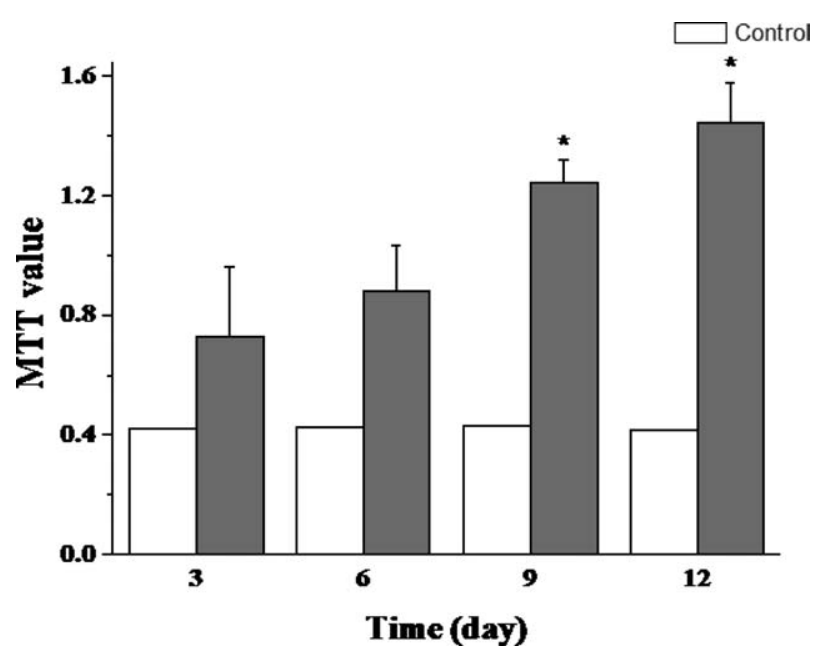

FIGURE 10. The chondrocytes viability when cultured in Col/Cht-50 scaffolds. * indicates statistical significance when compared with MTT values of day 3 and day $6(p<0.05)$

[Fig. 8(a)]. Furthermore, Figure 8(a) shows that $E^{\prime}$ increased from $2.4 \times 10^{5}$ to $4.6 \times 10^{5} \mathrm{~Pa}$ when chitosan amount increased from 0 to $50 \%$. However, although it showed an initial higher value in $E^{\prime}$, Col/Cht-50 presented less increase in $E^{\prime}$ as compared to Col/Cht-10 and Col/Cht-25 with the increasing frequency. From Figures 1 and 2 we observed that the pore size increase with the increase of chitosan amount and Figure 4 shows that a higher swelling ratio was verified for Col/Cht-50 but, in Figure 3 a higher cross-linking degree was verified. Moreover, the larger pore wall and the ordered honeycomb-like structure of Col/Cht-50 can lead to a retention of PBS in the pores where the deformability of the scaffolds are more difficult due to a hydrostatic effect leading to a not so pronounced increase in $E^{\prime}{ }^{44}$

The influence of chitosan amount on the loss factors $(\tan \delta$ ) is presented in Figure $8(\mathrm{~b})$. Values typically higher than 0.2 were observed, evidencing the clear viscoelastic nature of the structures. It was observed that Col/Cht-10 and Col/Cht-25 presented similar behaviors of $\tan \delta$ which were distinct from that of $\mathrm{Col}$ and Col/Cht-50. Tan $\delta$ of Col slightly decreased with the increase of frequency indicating its lower viscosity as compared to other groups. Tan $\delta$ values of Col/Cht-10 and Col/Cht-25 increased with frequency indicating their higher damping capabilities than that of the other two groups. The $\tan \delta$ of Col/Cht-50 only increased above $1 \mathrm{~Hz}$. The large pore wall and ordered honeycomblike structure of Col/Cht-50 led to its superior PBS retention ability confirmed in Figure 4. Here the hydrostatic effect of water entrapped within the pores lead to an increase of the values of $\tan \delta .^{44}$

The variation of the viscoelastic properties by increasing the genipin concentration for Col/Cht-50 scaffolds is presented in Figure 9. In Figure 9(a), we can observe that $E^{\prime}$ increase with the increase of genipin concentration $(\approx 2.7 \times$ $10^{5}$ to $6.3 \times 10^{5}$ for $f=0.1 \mathrm{~Hz}$ ) promoting the stiffness of the scaffolds. ${ }^{45,46}$ It is shown in Figure 2 that no noticeable changes were observed in the morphologies and pore sizes of the scaffolds by increasing the genipin concentration. In Figure 3, an increase on the cross-linking degree was observed by the increase in genipin concentration from 0.1 to $0.5 \%$. Moreover, the swelling ratio was slightly decreased with the increase of genipin concentration. All these data are in agreement with the DMA results of Col/Cht-50. Figure 9(b) represents the variation of the loss factor $(\tan \delta)$ along the frequency. The loss factor is the ratio of the amount of energy dissipated by viscous mechanisms relative to energy stored in the elastic component providing information about the damping properties of the material. For all formulations, $\tan \delta$ increases with the increase of frequency indicating that the materials became more viscous and less elastic. Since all the formulations are only different in the genipin concentration, the values of $\tan \delta$ are very similar to all although, $0.1 \%$ genipin presented slightly higher values, which is ascribed to the lower cross-linking degree. ${ }^{47}$

\section{Cell viability and cell morphology}

In this study, a MTT assay was carried out to measure the relative viability of chondrocytes. Figure 10 displays the
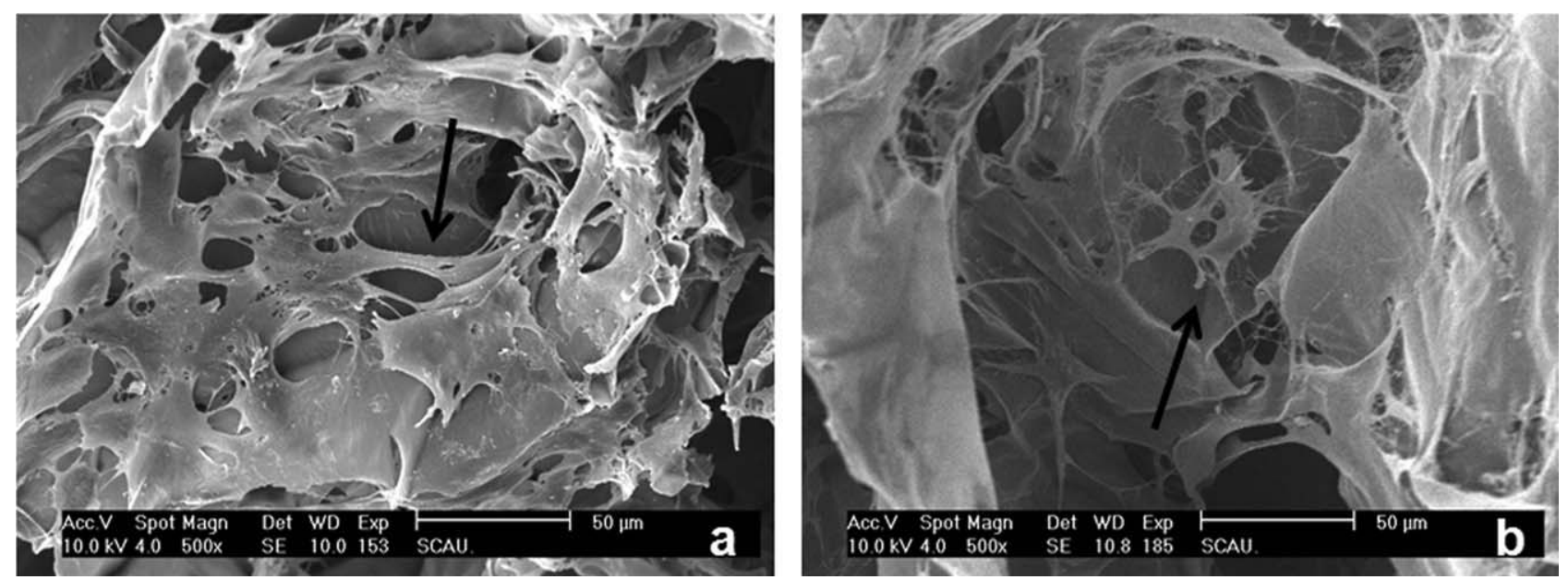

FIGURE 11. Morphology of chondrocytes seeded onto the surface of Col/Cht-50 scaffolds after 12 days of culturing, (a) on the surface of the scaffold; (b) inside the scaffold. 
viability of chondrocytes seeded onto the $0.3 \%$ genipin cross-linked Col/Cht-50 scaffolds. There was a significant improvement in MTT value as prolonging the culture time. The MTT values were increased from 0.726 (day 3) to 1.446 (day 12). The increase of MTT value between day 6 and day 9 was the highest during the tested adjacent two time periods. The continuous increasing of MTT values can be related to the scaffolds noncytotoxic behavior and proved that genipin treatment did not induce any deleterious effect on cells viability. MTT values of the controls were nearly unchanged and remained at a low level (Fig. 10). Thereby, the blue color of the scaffolds introduced by genipin treatment is stable and will not affect the MTT test. These results revealed that the scaffolds were noncytotoxic and benefitted the viability of chondrocytes.

Figure 11 shows SEM images of chondrocytes grown in the Col/Cht-50 scaffold after culturing for 12 days. It can be observed that the cells spread on the surface of the pore walls in the scaffold. The cells contacted each other by the extended lamellipodial protrusions, which is an indication of cell's activation. It can be observed that the chondrocytes presented fibroblastic-like morphology [black arrows in Fig. 11(a,b)] just as the morphology displayed in the 2D culture in the cell culture plates before seeding in the scaffold (data not shown). This image supports the idea that genipincross-linked collagen/chitosan scaffolds promoted the attachment and viability of chondrocytes and may serve as a suitable 3D matrix for cartilage tissue engineering applications.

\section{CONCLUSIONS}

In this study, we perform the physicochemical characterization and preliminary assessment of the biological performance of genipin-cross-linked collagen/chitosan porous scaffolds. The SEM images confirmed that the pore size and the morphology of the scaffolds can be tailored by the varying the amount of chitosan. Supported by the swelling data, we found that the cross-linking treatment didn't decrease the PBS retention abilities of the collagen/chitosan scaffolds. In vitro biodegradation demonstrated that the biostability of the collagen in the cross-linked collagen/chitosan scaffolds was improved. All the prepared collagen/chitosan porous scaffolds presented elastic properties during DMA test; in the case of Col/Cht-50, the elasticity modulus increased with the increasing of genipin concentration. The in vitro cell culture study demonstrated that the scaffolds displayed suitable biocompatibility as it can support chondrocyte's adhesion, spread onto the surface and the inside of the scaffold and, as well as chondrocyte's viability. Therefore, the genipin-cross-linked collagen/chitosan may be an interesting formulation for further study as scaffolding in articular cartilage tissue engineering.

\section{ACKNOWLEDGMENTS}

The authors thank Prof. Sung Hsing-Wen and his Ph.D. student Kokola (National Tsing Hua University, Taiwan) for their help in the ninhydrin assay, Prof. Li Hong (Jinan University), Mr. Albino Martins, Dr. Song W.L. (University of Minho) for their helpful suggestion to this work.

\section{REFERENCES}

1. Mano JF, Reis RL. Osteochondral defects: Present situation and tissue engineering approaches. J Tissue Eng Regen Med 2007;1: 261-273.

2. Oliveira JM, Rodrigues MT, Silva SS, Malafaya PB, Gomes ME, Viegas CA, Dias IR, Azevedo JT, Mano JF, Reis RL. Novel hydroxyapatite/chitosan bilayered scaffold for osteochondral tissueengineering applications: Scaffold design and its performance when seeded with goat bone marrow stromal cells. Biomaterials 2006;27:6123-6137.

3. Fan $\mathrm{HB}$, Hu YY, Zhang $\mathrm{CL}$, Li XS, Lv R, Qin L, Zhu R. Cartilage regeneration using mesenchymal stem cells and a PLGA-gelatin/ chondroitin/hyaluronate hybrid scaffold. Biomaterials 2006;27: 4573-4580.

4. Shao XX, Hutmacher DW, Ho ST, Goh JCH, Lee EH. Evaluation of a hybrid scaffold/cell construct in repair of high-load-bearing osteochondral defects in rabbits. Biomaterials 2006;27:1071-1080.

5. Marsano A, Millward-Sadler SJ, Salter DM, Adesida A, Hardingham T, Tognana E, Kon E, Chiari-Grisar C, Nehrer S, Jakob M, Martin I. Differential cartilaginous tissue formation by human synovial membrane, fat pad, meniscus cells and articular chondrocytes. Osteoarthr Cartilage 2007;15:48-58.

6. Langer R, Vacanti JP. Tissue engineering. Science 1993;260: 920-926.

7. Hutmacher DW. Scaffolds in tissue engineering bone and cartilage. Biomaterials 2000;21:2529-2543.

8. Lee JE, Kim KE, Kwon IC, Ahn HJ, Lee SH, Cho HC, Kim HJ, Seong SC, Lee MC. Effects of the controlled-released TGF-beta 1 from chitosan microspheres on chondrocytes cultured in a collagen/chitosan/glycosaminoglycan scaffold. Biomaterials 2004;25: 4163-4173.

9. Zhen Li, Shan-Jing Yao, Mauro Alini, Stoddart MJ. Chondrogenesis of human bone marrow mesenchymal stem cells in fibrin-polyurethane composites is modulated by frequency and amplitude of dynamic compression and shear stress. Tissue Eng Part A 2010;16:575-584.

10. Oliveira JT, Crawford A, Mundy JM, Moreira AR, Gomes ME, Hatton PV, Reis RL. A cartilage tissue engineering approach combining starch-polycaprolactone fibre mesh scaffolds with bovine articular chondrocytes. J Mater Sci Mater Med 2007;18:295-302.

11. Moutos FT, Freed LE, Guilak F. A biomimetic three-dimensional woven composite scaffold for functional tissue engineering of cartilage. Nat Mater 2007;6:162-167.

12. Malafaya PB, Pedro AJ, Peterbauer A, Gabriel C, Redl H, Reis RL. Chitosan particles agglomerated scaffolds for cartilage and osteochondral tissue engineering approaches with adipose tissue derived stem cells. J Mater Sci-Mater Med 2005;16:1077-1085.

13. Iwasaki N, Yamane ST, Majima T, Kasahara Y, Minami A, Harada K, Nonaka S, Maekawa N, Tamura H, Tokura S, Shiono M, Monde K, Nishimura SI. Feasibility of polysaccharide hybrid materials for scaffolds in cartilage tissue engineering: Evaluation of chondrocyte adhesion to polyion complex fibers prepared from alginate and chitosan. Biomacromolecules 2004;5:828-833.

14. Griffon DJ, Sedighi MR, Schaeffer DV, Eurell JA, Johnson AL. Chitosan scaffolds: Interconnective pore size and cartilage engineering. Acta Biomater 2006;2:313-320.

15. Minas T, Nehrer S. Current concepts in the treatment of articular cartilage defects. Orthopedics 1997;20:525-538.

16. Hunter CJ, Imler SM, Malaviya $P$, Nerem RM, Levenston ME. Mechanical compression alters gene expression and extracellular matrix synthesis by chondrocytes cultured in collagen I gels. Biomaterials 2002;23:1249-1259.

17. Nehrer S, Breinan HA, Ramappa A, Shortkroff S, Young G, Minas T, Sledge CB, Yannas IV, Spector M. Canine chondrocytes seeded in type I and type II collagen implants investigated in vitro. J Biomed Mater Res 1997;38:95-104.

18. Wakitani S, Goto T, Young RG, Mansour JM, Goldberg VM, Caplan Al. Repair of large full-thickness articular cartilage defects with allograft articular chondrocytes embedded in a collagen gel. Tissue Eng 1998;4:429-444.

19. Lee $\mathrm{CH}$, Singla $\mathrm{A}$, Lee $\mathrm{Y}$. Biomedical applications of collagen. Int J Pharm 2001;221:1-22. 
20. Ma L, Gao CY, Mao ZW, Zhou J, Shen JC, Hu XQ, Han CM. Collagen/chitosan porous scaffolds with improved biostability for skin tissue engineering. Biomaterials 2003;24:4833-4841.

21. Weadock KS, Miller EJ, Bellincampi LD, Zawadsky JP, Dunn MG. Physical crosslinking of collagen fibers: Comparison of ultraviolet irradiation and dehydrothermal treatment. J Biomed Mater Res 2004;29:1373-1379.

22. Lynn AK, Yannas IV, Bonfield W. Antigenicity and immunogenicity of collagen. J Biomed Mater Res B Appl Biomater 2004;71: 343-354.

23. Liu BC, Harrell R, Davis $\mathrm{RH}$, Dresden MH, Spira M. The effect of gamma-irradiation on injectable human amnion collagen. J Biomed Mater Res 1989;23:833-844.

24. Ma L, Gao CY, Mao ZW, Shen JC, Hu XQ, Han CM. Thermal dehydration treatment and glutaraldehyde cross-linking to increase the biostability of collagen-chitosan porous scaffolds used as dermal equivalent. J Biomater Sci Polym Ed 2003;14:861-874.

25. Park SN, Park JC, Kim HO, Song MJ, Suh H. Characterization of porous collagen/hyaluronic acid scaffold modified by 1-ethyl-3-(3dimethylaminopropyl)carbodiimide cross-linking. Biomaterials 2002;23:1205-1212.

26. Chiono V, Pulieri E, Vozzi G, Ciardelli G, Ahluwalia A, Giusti P. Genipin-crosslinked chitosan/gelatin blends for biomedical applications. J Mater Sci Mater Med 2008;19:889-898.

27. Yuan Y, Chesnutt BM, Utturkar G, Haggard WO, Yang Y, Ong JL, Bumgardner JD. The effect of cross-linking of chitosan microspheres with genipin on protein release. Carbohydr Polym 2007; 68:561-567.

28. Huang LLH, Sung HW, Tsai CC, Huang DM. Biocompatibility study of a biological tissue fixed with a naturally occurring crosslinking reagent. J Biomed Mater Res 1998;42:568-576.

29. Liang HC, Chang WH, Lin KJ, Sung HW. Genipin-crosslinked gelatin microspheres as a drug carrier for intramuscular administration: In vitro and in vivo studies. J Biomed Mater Res A 2003;65: 271-282.

30. Mi FL, Tan YC, Liang HF, Sung HW. In vivo biocompatibility and degradability of a novel injectable-chitosan-based implant. Biomaterials 2002;23:181-191.

31. Silva SS, Motta A, Rodrigues MT, Pinheiro AFM, Gomes ME, Mano JF, Reis RL, Migliaresi C. Novel genipin-cross-linked chitosan/silk fibroin sponges for cartilage engineering strategies. Biomacromolecules 2008;9:2764-2774.

32. Malafaya PB, Santos TC, Van Griensven M, Reis RL. Morphology, mechanical characterization and in vivo neo-vascularization of chitosan particle aggregated scaffolds architectures. Biomaterials 2008;29:3914-3926.

33. Di Martino A, Sittinger M, Risbud MV. Chitosan: A versatile biopolymer for orthopaedic tissue-engineering. Biomaterials 2005;26: 5983-5990.

34. Sionkowska A, Wisniewski M, Skopinska J, Kennedy CJ, Wess TJ. Molecular interactions in collagen and chitosan blends. Biomaterials $2004 ; 25: 795-807$.
35. Sung H-W, Chang $\mathrm{Y}$, Chiu C-T, Chen C-N, Liang H-C. Mechanical properties of a porcine aortic valve fixed with a naturally occurring crosslinking agent. Biomaterials 1999;20:1759-1772.

36. Pieper JS, Oosterhof A, Dijkstra PJ, Veerkamp JH, Van Kuppevelt $\mathrm{TH}$. Preparation and characterization of porous crosslinked collagenous matrices containing bioavailable chondroitin sulphate. Biomaterials 1999;20:847-858.

37. Woessner JF. The determination of hydroxyproline in tissue and protein samples containing small proportions of this imino acid. Arch Biochem Biophys 1961;93:440-447.

38. Sung HW, Huang RN, Huang LLH, Tsai CC, Chiu CT. Feasibility study of a natural crosslinking reagent for biological tissue fixation. J Biomed Mater Res 1998;42:560-567.

39. Mi FL, Sung HW, Shyu SS. Synthesis and characterization of a novel chitosan-based network prepared using naturally occurring crosslinker. J Polym Sci Polym Chem 2000;38:2804-2814.

40. Dahlberg L, Billinghurst RC, Manner P, Nelson F, Webb G, Ionescu M, Reiner A, Tanzer M, Zukor D, Chen J, Van Wart HE, Poole AR. Selective enhancement of collagenase-mediated cleavage of resident type II collagen in cultured osteoarthritic cartilage and arrest with a synthetic inhibitor that spares collagenase 1 (matrix metalloproteinase 1). Arthritis Rheum 2000;43:673-682.

41. Mano JF. Viscoelastic properties of chitosan with different hydration degrees as studied by dynamic mechanical analysis. Macromol Biosci 2008;8:69-76.

42. Mano JF, Reis, RL, Cunha AM. Dynamic mechanical analysis in polymers for medical applications. In: Reis RL, Cohn D, editors. Polymer Based Systems on Tissue Engineering, Replacement and Regeneration, Vol. 86. Netherlands: Kluwer Academic Publishers, 2002. p 139-164.

43. Mano JF, Neves NM, Reis RL. Mechanical characterization of biomaterials. In: Reis RL, Román JS, editors. Biodegradable Systems in Tissue Engineering and Regenerative Medicine. CRC Press, 2005.

44. Ghosh S, Gutierrez V, Fernandez C, Rodriguez-Perez MA, Viana JC, Reis RL, Mano JF. Dynamic mechanical behavior of starchbased scaffolds in dry and physiologically simulated conditions: Effect of porosity and pore size. Acta Biomater 2008;4:950-959.

45. Silva RM, Silva GA, Coutinho OP, Mano JF, Reis RL. Preparation and characterisation in simulated body conditions of glutaraldehyde crosslinked chitosan membranes. J Mater Sci-Mater Med 2004;15:1105-1112.

46. Caridade SG, da Silva RMP, Reis RL, Mono JF. Effect of solventdependent viscoelastic properties of chitosan membranes on the permeation of 2-phenylethanol. Carbohydr Polym 2009;75: 651-659.

47. Alves NM, Ribelles JLG, Tejedor JAG, Mano JF. Viscoelastic behavior of poly(methyl methacrylate) networks with different cross-linking degrees. Macromolecules 2004;37:3735-3744. 